\title{
Chemical identification and bond control of $\pi$-skeletons in a coupling
}

\section{reaction}

Chi Zhang ${ }^{1}$, Rafael B. Jaculbia ${ }^{1}$, Yusuke Tanaka ${ }^{2,3}$, Emiko Kazuma ${ }^{1}$, Hiroshi Imada ${ }^{1}$, Norihiko Hayazawa $^{1}$, Atsuya Muranaka ${ }^{3}$, Masanobu Uchiyama ${ }^{2,3}$, and Yousoo Kim ${ }^{1 *}$

${ }^{1}$ Surface and Interface Science Laboratory, RIKEN, 2-1 Hirosawa, Wako, Saitama 351-0198, Japan ${ }^{2}$ Graduate School of Pharmaceutical Sciences, The University of Tokyo, 7-3-1 Hongo, Bunkyo-ku, Tokyo 113-0033, Japan

${ }^{3}$ Advanced Elements Chemistry Laboratory, RIKEN, 2-1 Hirosawa, Wako, Saitama 351-0198, Japan

"E-mail: ykim@riken.jp

\section{Supporting information}

\section{Table of contents:}

Methods

Synthetic procedures

Supplementary STM images, STS, TERS spectra, Raman spectrum, and DFT calculations

References 


\section{Methods}

All the STM/STS experiments were performed with a low-temperature (LT) STM system, from Omicron $\mathrm{GmbH}$, maintained at $\sim 5 \mathrm{~K}$ under ultrahigh vacuum conditions with a base pressure of $\sim 4.0 \times 10^{-11}$ Torr, and electrochemically etched W tips were used. STS measurements were conducted using standard lock-in techniques with bias modulation of $30 \mathrm{mV}$ and frequency of 797 Hz. The STM-TERS experiments were performed with another Omicron LT-STM system maintained at $<4.8 \mathrm{~K}$ with a base pressure of $\sim 1.0 \times 10^{-11}$ Torr, and electrochemically etched $\mathrm{Au}$ tips.

Sample preparation. The $\mathrm{Ag}(111)$ and $\mathrm{Ag}(110)$ substrates were cleaned by $\mathrm{Ar}^{+}$-ion sputtering and annealing at $\sim 860 \mathrm{~K}$ for repeated cycles. DEBP and DPBD with purity exceeding $98.0 \%$ were purchased from TCI Co., and thoroughly degassed before deposition. The 1,3-enyne molecule, was synthesized ex situ in solution (as detailed in the synthetic procedures below) according to the reported reaction routes ${ }^{1}$, and also thoroughly degassed. DEBP and DPBD were deposited through leak valves, while the 1,3-enyne was evaporated using a home-made Knudsen-cell at $\sim 450 \mathrm{~K}$. After deposition of the molecules onto the $\operatorname{Ag}(111)$ or $\operatorname{Ag}(110)$ substrate, we transferred the sample to the STM head for further measurements. All the annealing procedures were performed at temperatures indicated in the figures, typically for $30 \mathrm{~min}$. WSxM and Gwyddion software packages were used for processing the STM images.

STM-TERS experiments. In the STM-TERS experiments, the STM junction was illuminated for more than two hours before data collection to minimize thermal effects. A Spectra Physics model 3900 s tunable titanium sapphire laser set to $738 \mathrm{~nm}$ was used for excitation, and p-polarized laser was introduced with appropriate optics into the STM junction. An aspheric lens positioned near the STM tip at an angle of $65^{\circ}$ from the surface normal was used to focus the incident laser on the STM junction. Raman signals were collected by an achromatic lens at the other side of the STM junction, and then passed through a long-pass filter into a Princeton Instruments Isoplane-320 spectrometer. A slit width of $50 \mu \mathrm{m}$ and grating of $600 \mathrm{~g} / \mathrm{mm}$ was used for all measurements. Several STM scans were performed under $V_{s}=0.1 \mathrm{~V}$ and $I_{t}=5 \mathrm{pA}$ conditions before TERS measurements to ensure the stability of the molecular system. Thereafter, the Au tip was placed at a target position, the feedback loop was turned off and a controlled tip approach was applied, typically of $0.5 \mathrm{~nm}$ (i.e., $\Delta \mathrm{Z}=-0.5 \mathrm{~nm}$ ). The exposure time was typically set to $3 \mathrm{~min}$ for each spectrum acquisition.

Statistics on the $\mathbf{C U} /$ total connection ratios. $\mathrm{CU} /$ total connection ratios were evaluated from examinations of non-repetitive areas of around $25000 \mathrm{~nm}^{2}$. For samples obtained with annealing temperatures of $\sim 310 \mathrm{~K}$, around 370 connections were counted in the areas (owing to the low yield of covalent connections), and the average percentage (mean $\pm \mathrm{SD}$ ) of $\mathrm{CU}$ connections was found to be $21.2 \pm 5.0 \%$. In samples obtained with higher annealing temperatures of $\sim 370 \mathrm{~K}$, more 
connections were formed, so around 2730 connections were counted, and CU connection were found to account for $8.6 \pm 0.6 \%$ of the total, on average.

DFT calculations. Most of the calculations were performed in the DFT framework using the Vienna ab initio simulation package (VASP). The projector-augmented wave method was used to describe the interactions between ions and electrons ${ }^{2,3}$. The Perdew-Burke-Ernzerhof generalized gradient approximation exchange-correlation functional was employed ${ }^{4}$, and van der Waals interactions were included using the dispersion-corrected DFT-D3 method of Grimme ${ }^{5}$. The atomic structures were relaxed using the conjugate gradient algorithm scheme as implemented in the VASP code until the forces on all unconstrained atoms were $\leqslant 0.02 \mathrm{eV} / \AA$. Plane waves were used as a basis set with an energy cutoff of $450 \mathrm{eV}$. $\mathrm{Ag}(111)$ substrate was modelled by four-layered slabs separated by a $\sim 20 \AA$ vacuum region for the structural models, where the bottom two layers were fixed. Simulated STM images were obtained based on the Tersoff-Hamann method $^{6}$. The climbing-image nudged elastic band (CI-NEB) was applied to locate the transition states ${ }^{7}$, and the transition paths were optimized until the forces acting on the path were $\leqslant 0.03 \mathrm{eV} / \AA$. Raman spectra were calculated using the Gaussian 16 program package. The hybrid functional PBE0 with a $6-31 \mathrm{G}(\mathrm{d}, \mathrm{p})$ basis set was applied for the vibrational frequency calculations. All the vibrational frequencies were scaled by a factor of 0.96 .

\section{Synthetic procedures}

General remarks. ${ }^{1} \mathrm{H}$ NMR spectra were recorded on a JEOL AL-400 NMR (400 MHz) spectrometer. All starting materials, reagents, and solvents were purchased from FUJIFILM Wako Pure Chemical Co., Sigma-Aldrich Inc., and other commercial suppliers and used as received. (E)4,4"-(but-1-en-3-yne-1,4-diyl)di-1,1'-biphenyl was synthesized according to the literature method ${ }^{1}$.

Synthesis of (E)-4,4"-(but-1-en-3-yne-1,4-diyl)di-1,1'-biphenyl. In a $20 \mathrm{~mL}$ Schlenk tube, a mixture of 4-ethynyl-1-1'-biphenyl (501 mg, $2.81 \mathrm{mmol})$, PEPPSI-IPr (21.5 mg, $0.0316 \mathrm{mmol}$ ), and potassium hydroxide $(6.8 \mathrm{mg}, 0.121 \mathrm{mmol})$ was stirred in toluene/water $(6.2 \mathrm{~mL} / 0.03 \mathrm{~mL})$ at $60{ }^{\circ} \mathrm{C}$ under argon atmosphere for 20 hours. After cooling to room temperature, the solvent was removed in reduced pressure. The residue was dissolved in dichloromethane $(300 \mathrm{~mL})$ and purified by short silica gel column chromatography. Evaporation of the solvent gave the desired product (480 mg, $1.35 \mathrm{mmol}, 96 \%) .{ }^{1} \mathrm{H}-\mathrm{NMR}\left(400 \mathrm{MHz}, \mathrm{CDCl}_{3}\right): \delta 7.64-7.36(\mathrm{~m}, 18 \mathrm{H}), 7.10(\mathrm{~d}, J=16.0$ $\mathrm{Hz}, 1 \mathrm{H}), 6.46(\mathrm{~d}, J=16.0 \mathrm{~Hz}, 1 \mathrm{H})$; LRMS (MALDI, positive) $m / z$ calcd for $\mathrm{C}_{28} \mathrm{H}_{20}[\mathrm{M}]^{+}: 356.1565$, found 356.2. The observed chemical shifts were in good agreement with those reported previously ${ }^{8}$. 


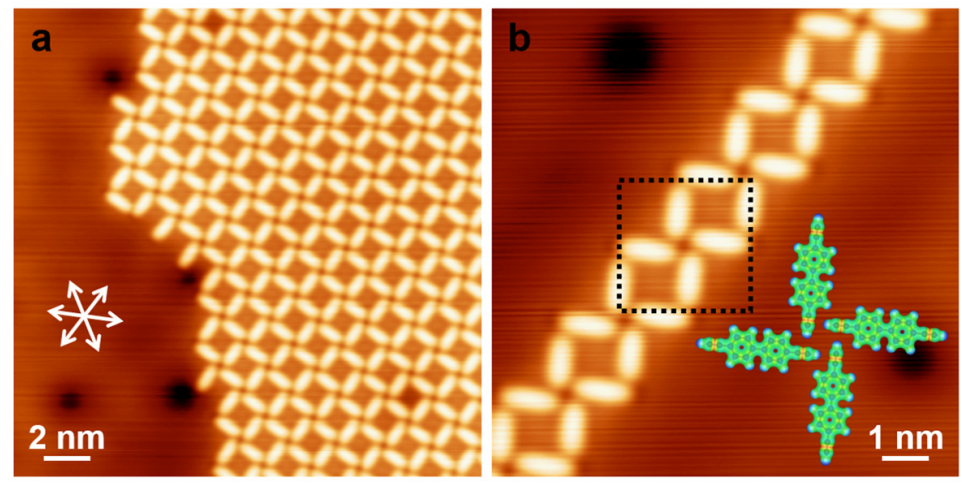

Figure S1. Formation of (a) molecular islands and (b) chains after deposition of DEBP onto $\operatorname{Ag}(111)$ held at room temperature. Scanning conditions: $V_{s}=0.1 \mathrm{~V}, I_{t}=0.1 \mathrm{nA}$. The inset in (b) shows the electrostatic potential map of one windmill-like motif indicating the $\mathrm{C}-\mathrm{H} \cdots \pi$ intermolecular interactions, where red and blue colors represent negative and positive potential regions, respectively.
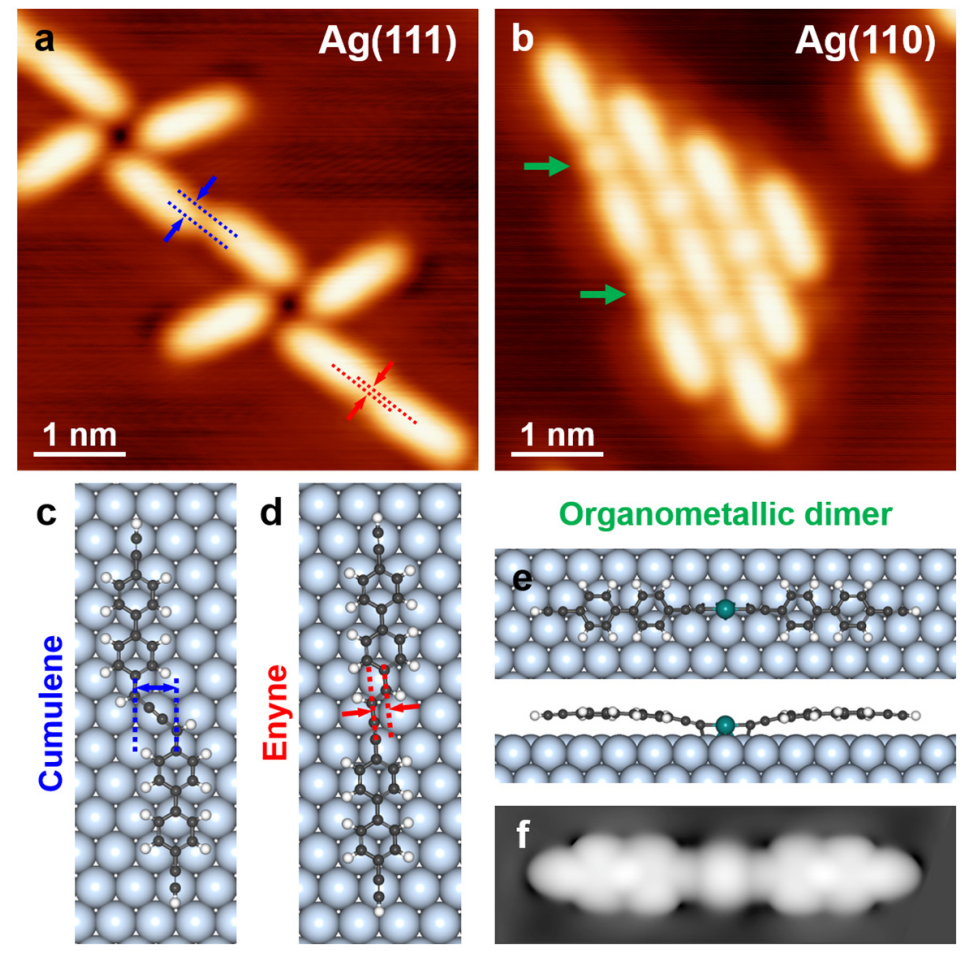

Figure S2. Comparison among EN, CU connections and potential organometallic connections. (a) STM image showing coexistence of DEBP, EN and CU dimers on $\mathrm{Ag}(111)$ after annealing. The 
offsets between neighboring units in EN and CU dimers are indicated by blue and red dotted lines and arrows, respectively. (b) STM image showing formation of dominant organometallic oligomers packed together on $\mathrm{Ag}(110)$ after annealing. The green arrows indicate Ag adatoms appearing as pronounced round protrusions involved in the oligomers. (c) and (d) DFT optimized structural models of (c) cumulene and (d) enyne dimers on $\operatorname{Ag}(111)$ with offsets indicated in blue and red, respectively. (e) DFT optimized structural model of a potential organometallic dimer on $\operatorname{Ag}(111)$ and (f) the corresponding simulated STM image.

The organometallic oligomers formed on $\operatorname{Ag}(110)$ agree well with the calculated dimer on $\operatorname{Ag}(111)$ in both morphology and dimension. Nevertheless, such organometallic structures were not observed on $\operatorname{Ag}(111)$ in the experimental conditions. Moreover, the linear configuration together with the protrusion feature between neighboring units in organometallic oligomers is inconsistent with the obvious offsets and seamless and smooth linkages in EN and CU connections. Therefore, the possibility of organometallic connections can be excluded for the assignment of either EN or CU.
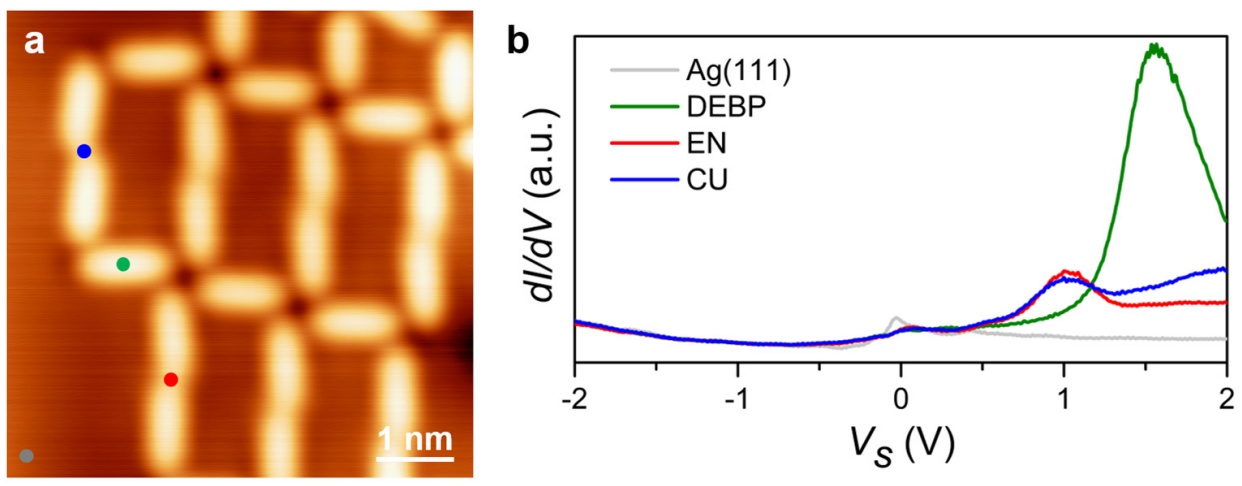

Figure S3. (a) STM image of molecular islands composed of DEBP monomer, EN and CU dimers. (b) $\mathrm{dI} / \mathrm{dV}$ spectra collected at the bare $\mathrm{Ag}(111)$, center of DEBP, EN and CU connections, as indicated in (a), respectively. 

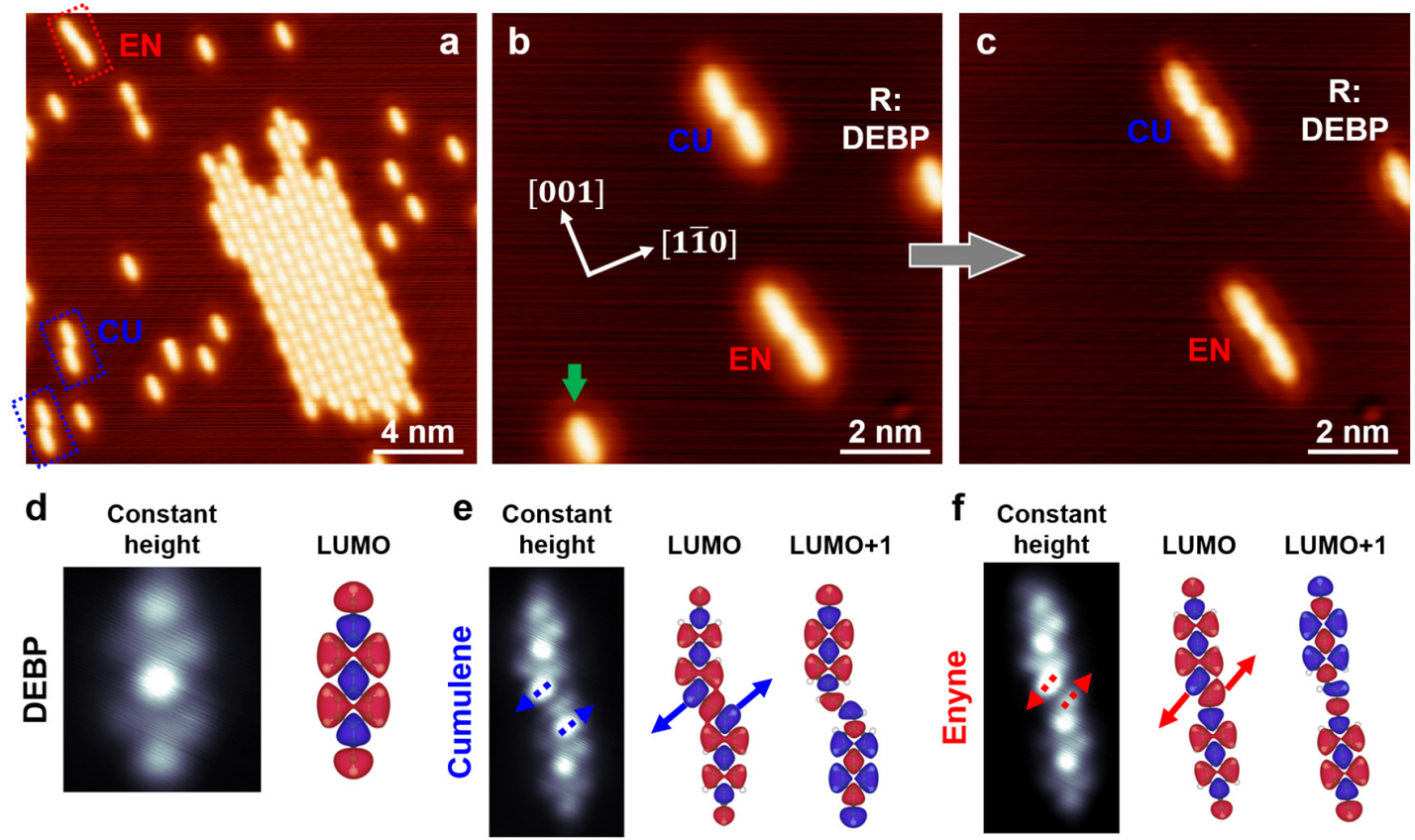

Figure S4. (a) Large-scale STM image showing the formation of dominant organometallic chains packed together and some isolated DEBP monomers, CU and EN dimers after deposition of DEBP onto $\mathrm{Ag}(110)$ at RT followed by annealing at $\sim 350 \mathrm{~K}$. (b) and (c) STM images obtained (b) before and (c) after picking up a DEBP molecule (as indicated by the green arrow) for functionalizing the tip apex. Scanning conditions: $V_{s}=0.1 \mathrm{~V}, I_{t}=1.0 \mathrm{nA}$. Typical conditions for picking up a DEBP are to set $V_{s}=0.01 \mathrm{~V}, I_{t}=48 \mathrm{nA}$ when the tip reaches the target molecule. The situation of a DEBPterminated tip is expected to be similar to that of a pentacene-tip which corresponds to an $s$-wave tip as reported ${ }^{9-11}$. (d)-(f) Left panels: constant height STM images of (d) DEBP, (e) cumulene and (f) enyne dimers obtained with a DEBP-functionalized tip. Right panels: the calculated LUMO of planar DEBP, LUMO and LUMO+1 of cumulene and enyne dimers in the gas phase, respectively. The arrows in (e) and (f) indicate the orientations of $\mathrm{C}\left(\mathrm{sp}^{2}\right)-\mathrm{H}\left(\sigma_{\mathrm{CH}}\right)$ lobes involved at the centers.

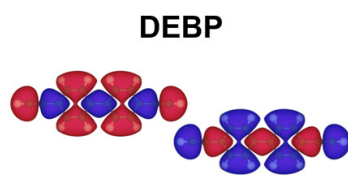

LUMO

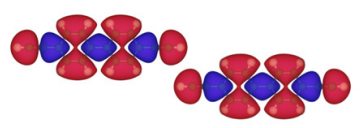

Enyne
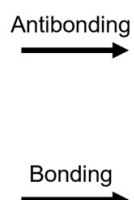
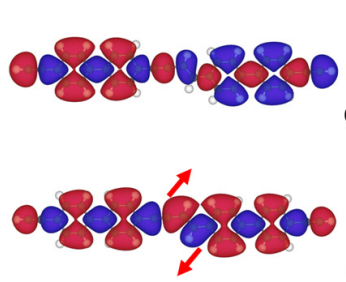

Cumulene

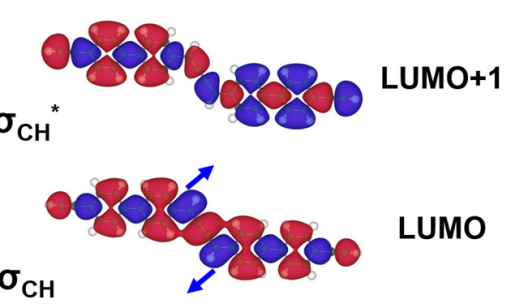


Figure S5. Calculated LUMO of DEBP, LUMO and LUMO+1 of enyne and cumulene dimers. The antibonding combination of two DEBP molecules results in the LUMO+1 of enyne and cumulene dimers with the $\sigma_{\mathrm{CH}}{ }^{*}$ lobes, while the bonding combination leads to the LUMO of enyne and cumulene featuring the $\sigma_{\mathrm{CH}}$ lobes. The orientations of the $\sigma_{\mathrm{CH}}$ lobes in enyne and cumulene are pointed out by the red and blue arrows, respectively.

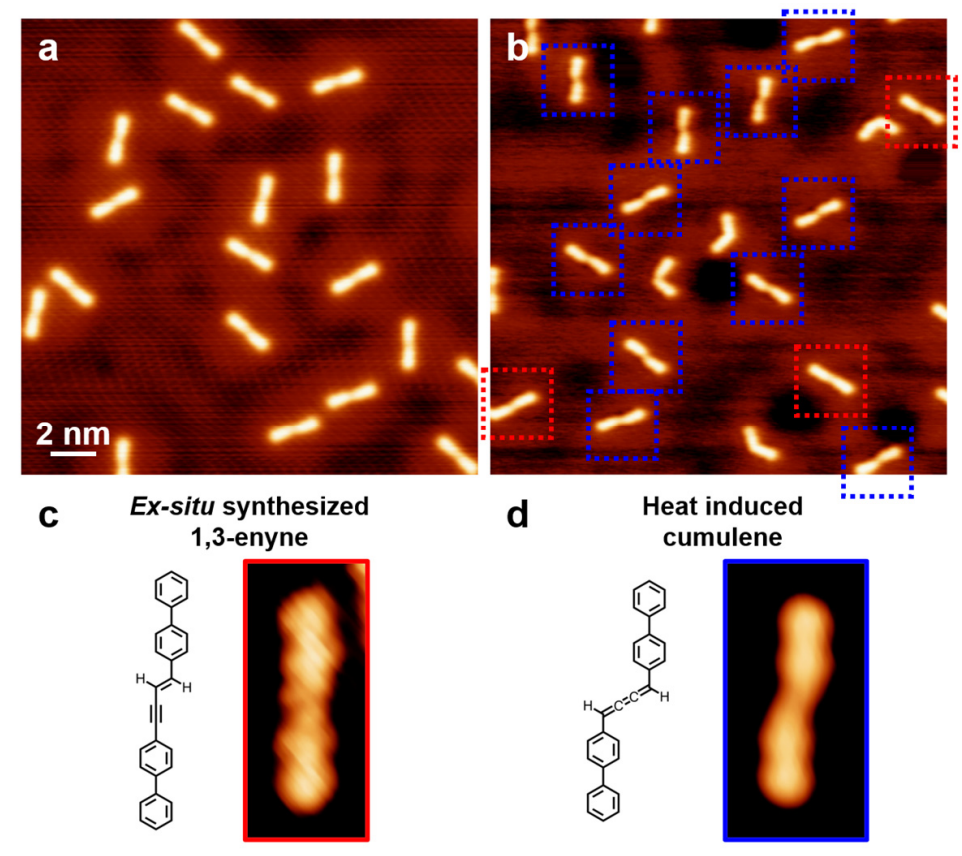

Figure S6. Ex-situ-synthesized 1,3-enyne deposited onto $\operatorname{Ag}(111)$ and heat-induced formation of cumulene from 1,3-enyne. (a) STM image showing dispersed 1,3-enyne molecules after deposition onto $\mathrm{Ag}(111)$ at RT. (b) STM image showing formation of cumulene coexisting with 1,3-enynes (as indicated by blue and red rectangles, respectively) after annealing at $\sim 420 \mathrm{~K}$. (c) and (d) Chemical structures (left panels) and corresponding STM images (right panels) of (c) the ex-situsynthesized 1,3-enyne and (d) heat-induced cumulene, respectively. 

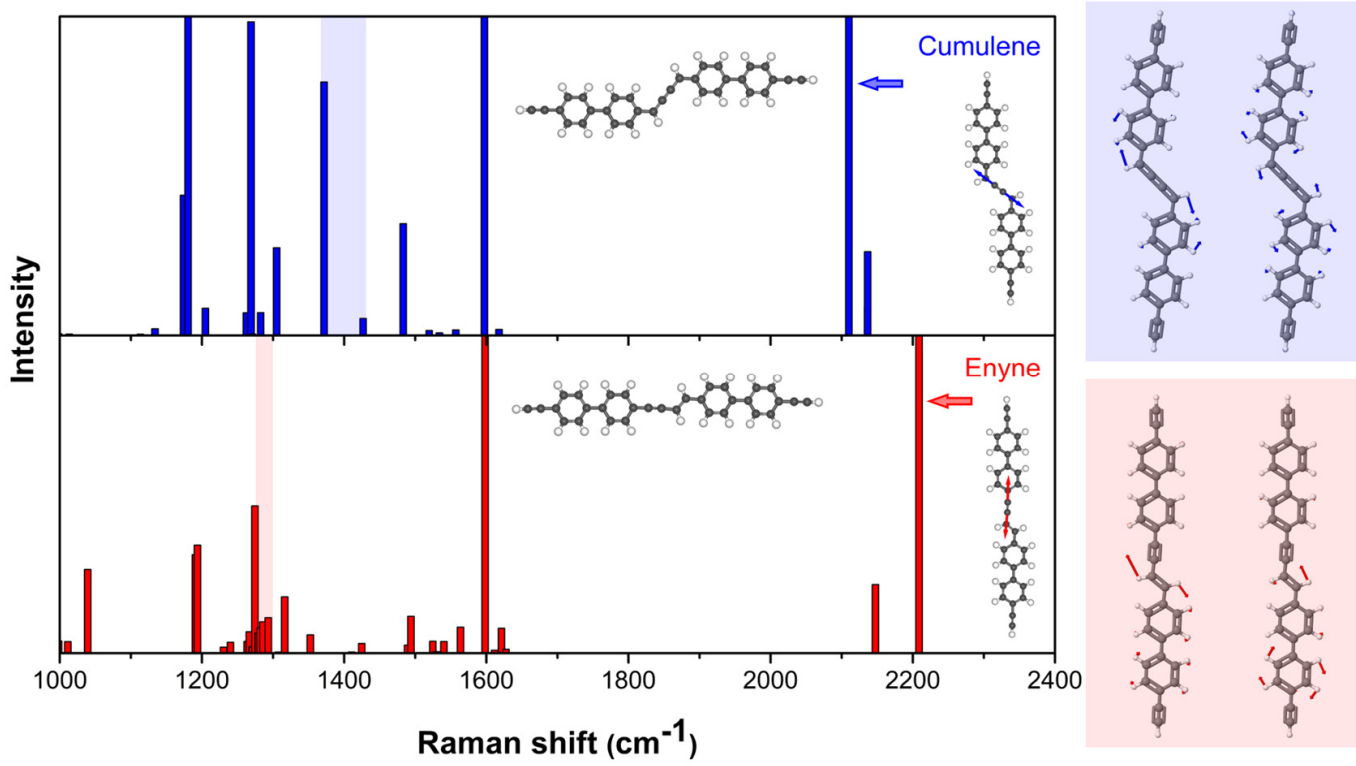

Figure S7. Calculated orientationally averaged Raman spectra of a planar cumulene (upper panel) and an enyne (lower panel) dimer in the gas phase. The calculated $\mathrm{C} \equiv \mathrm{C}$ stretching modes in the centers of cumulene and enyne dimers are indicated by the blue and red arrows, respectively. The other one present in the Raman-silent region stems from the terminal alkynyl group in both dimers. The characteristic $\mathrm{CH}$ bending in cumulene connection is calculated to be $1372 \mathrm{~cm}^{-1}, 1427 \mathrm{~cm}^{-1}$ as highlighted in the light blue range and shown in the right panel, while the calculated $\mathrm{CH}$ bending in enyne appears below $1300 \mathrm{~cm}^{-1}$ in the light red range (typically shown in the right panel).

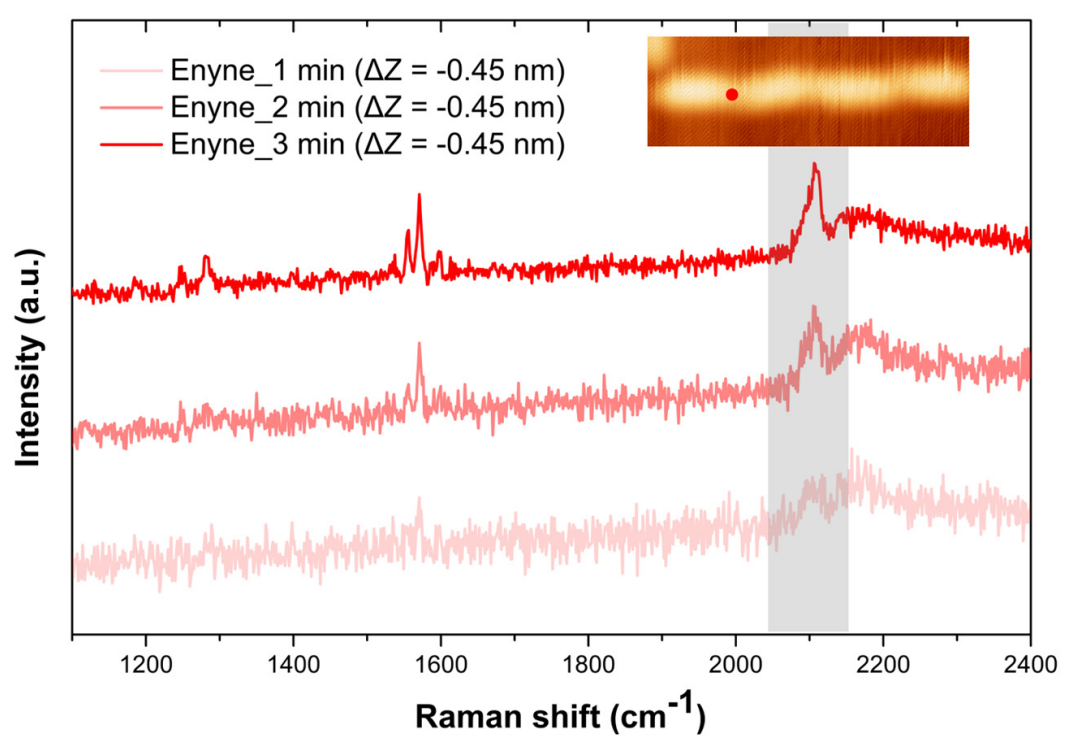


Figure S8. STM-TERS spectra collected for different exposure time (ranging from $1 \mathrm{~min}$ to $3 \mathrm{~min}$ ) when the tip was placed above the first connection (enyne) of the tetramer shown in the inset with $\Delta \mathrm{Z}=-0.45 \mathrm{~nm} . \Delta \mathrm{Z}$ is the tip height offset with respect to the setpoint of $V_{s}=0.1 \mathrm{~V}, I_{t}=5 \mathrm{pA}$ above the molecule. The spectra are vertically shifted for clarity.

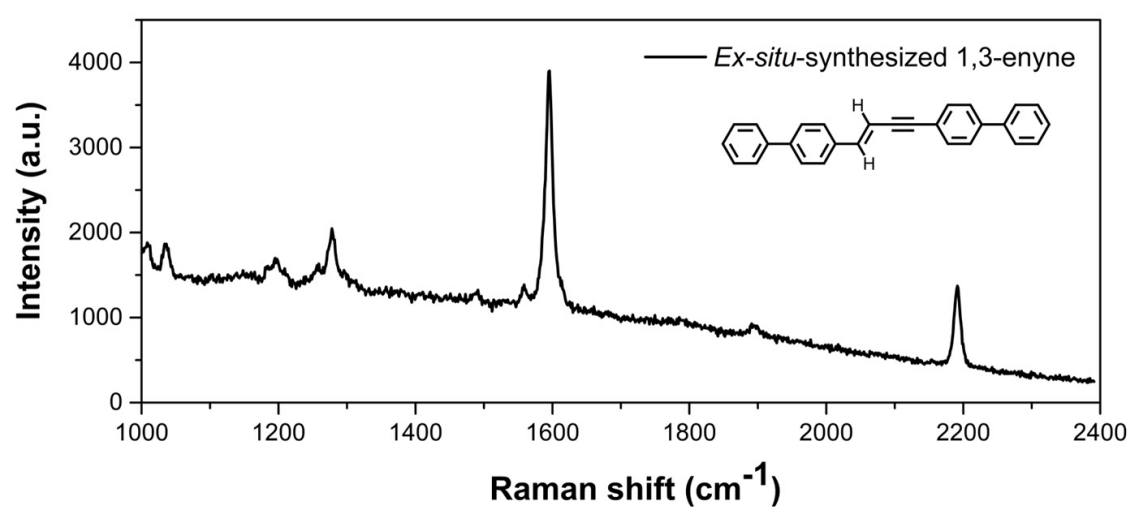

Figure S9. Raman spectrum of the ex-situ-synthesized 1,3-enyne molecule which was dissolved in acetone and further dropped onto a polycrystalline Au wire followed by drying in air. A laser set to $782 \mathrm{~nm}$ was used for excitation. The power was set to $\sim 1 \mathrm{~mW}$, and the exposure time was $60 \mathrm{~s}$. A grating of $600 \mathrm{~g} / \mathrm{mm}$ was used for the measurements.

Strong fluorescence was observed when we measured Raman spectrum of the ex-situ-synthesized 1,3-enyne molecule both in powder and in solution. We then tried to suppress the fluorescence and enhance the Raman signals. The ex-situ-synthesized 1,3-enyne molecule was dissolved in acetone, and the solution was further dropped onto a polycrystalline Au wire followed by drying in air. Due to the interaction between the enyne molecule and Au surface, the fluorescence was greatly suppressed, and we were able to obtain the Raman signals. Note that the ex-situ-synthesized enyne has no terminal alkynyl groups compared to the in-situ-synthesized ones on $\operatorname{Ag}(111)$. The result basically shows a good agreement with the calculated Raman spectra of a planar enyne (in-situsynthesized) dimer in the gas phase, and the $\mathrm{C} \equiv \mathrm{C}$ stretching modes of terminal alkynyl groups are naturally not involved in the experimental result as expected. 


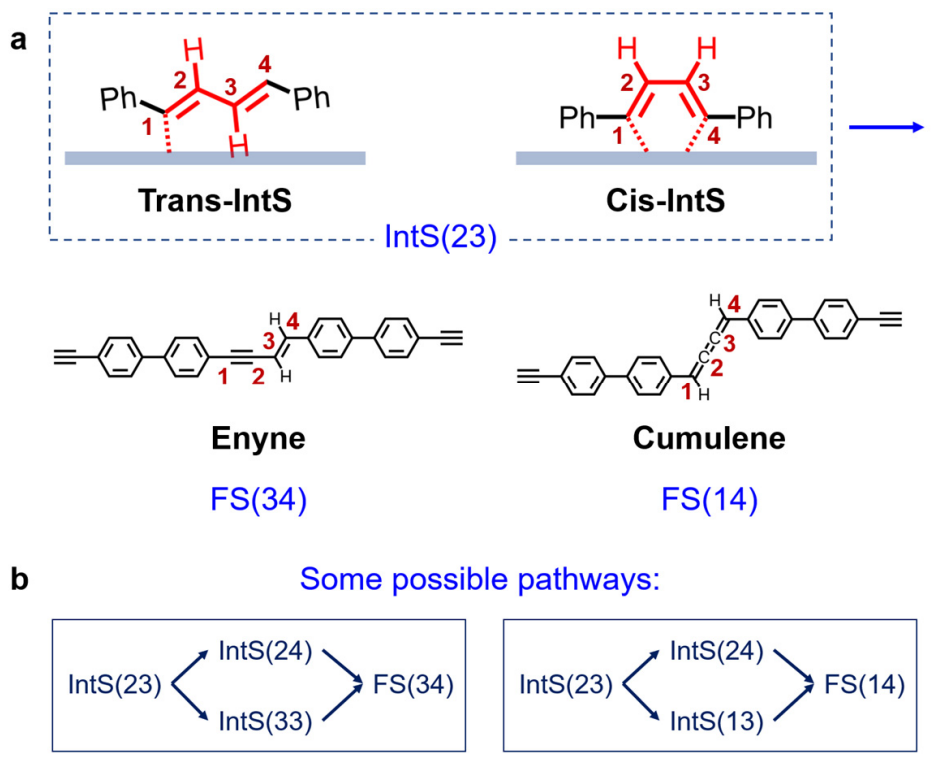

Figure S10. Schematic illustration showing some possible reaction pathways from the intermediate states (IntS23, either Cis-IntS or Trans-IntS) to enyne (FS34) and cumulene (FS14). The numbers here denote the hydrogen sites within the corresponding molecules. IntS: intermediate state; FS: final state.

As for the reaction mechanism of the non-dehydrogenative addition reaction of terminal alkynes, two intermediate states (IntS) are expected to be involved in the coupling reaction pathway, i.e., Trans-IntS and Cis-IntS as also reported before ${ }^{12}$. Furthermore, tautomerization processes may take place leading to the generation of enyne (i.e., FS(34)) and cumulene (i.e., FS(14)) connections. Therefore, two main processes are possible: (1) two DEBP $\rightarrow$ Trans-IntS $\rightarrow$ Cis-IntS $\rightarrow$ FS(34) or FS(14); and (2) two DEBP $\rightarrow$ Trans-IntS $\rightarrow$ FS(34) or FS(14). Note that the numbers here denote the hydrogen sites within the corresponding molecules to clearly show the involved tautomerization processes.

In the case of enyne formation, tautomerization processes may include possibilities of (1) IntS(23) $\rightarrow \operatorname{IntS}(24) \rightarrow \mathrm{FS}(34)$, and (2) $\operatorname{IntS}(23) \rightarrow \operatorname{IntS}(33) \rightarrow \mathrm{FS}(34)$. As for cumulene formation, the following processes may be involved: (1) $\operatorname{IntS(23)~} \rightarrow \operatorname{IntS}(24) \rightarrow \mathrm{FS}(14)$, and (2) $\operatorname{IntS}(23) \rightarrow$ IntS(13) $\rightarrow \mathrm{FS}(14)$. In addition, metal atoms have been reported to be able to reduce reaction barriers and play an important role in reaction processes ${ }^{13,14}$. As Ag adatoms are available on the substrate in the experimental conditions, the corresponding pathways involving $\mathrm{Ag}$ adatoms are also possible which will be a topic of interest to be explored in the future study. 


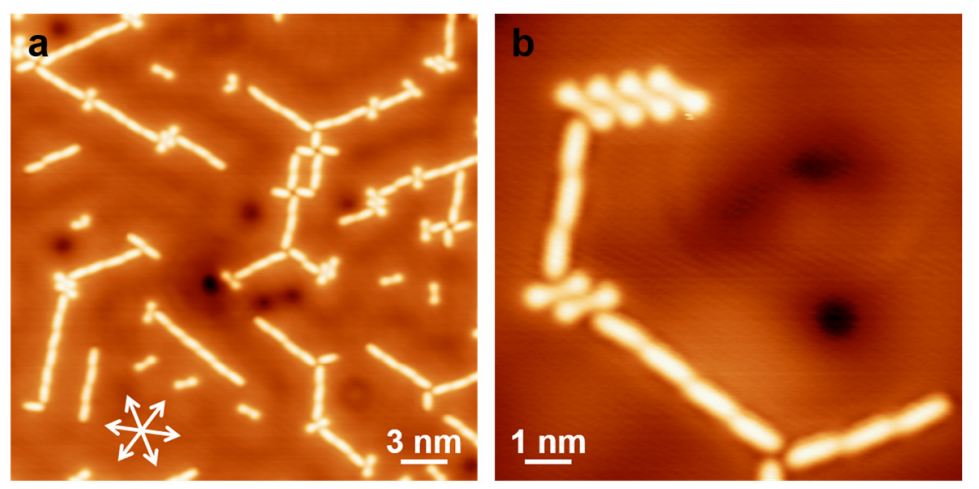

Figure S11. (a) Large-scale and (b) magnified STM images showing the aggregation among DEBP-based oligomers and DPBD molecules after annealing at $\sim 340 \mathrm{~K}$. The terminal alkynyl groups of DEBP-based oligomers always interact with the center (i.e., diyne skeleton) of DPBD molecules. Moreover, DPBD molecules interact with each other in a head-to-center mode showing the interaction between phenyl rings and central diyne skeletons. All the above intermolecular alignments stem from the $\mathrm{C}-\mathrm{H} \cdots \pi$ interactions similar to that shown in the DEBP windmill-like motifs. 


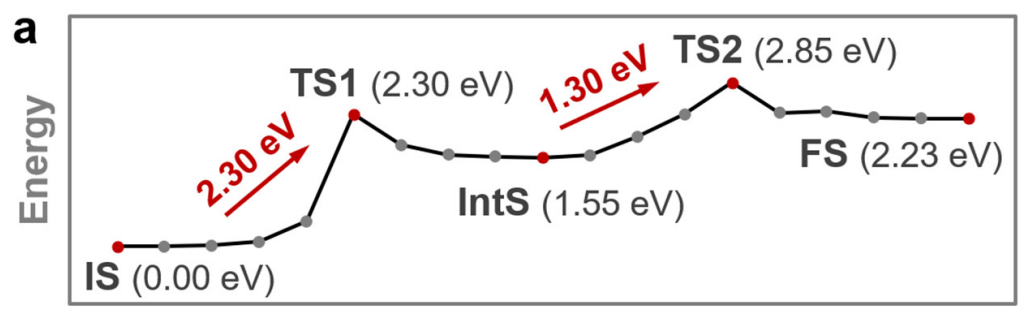

Reaction coordinate
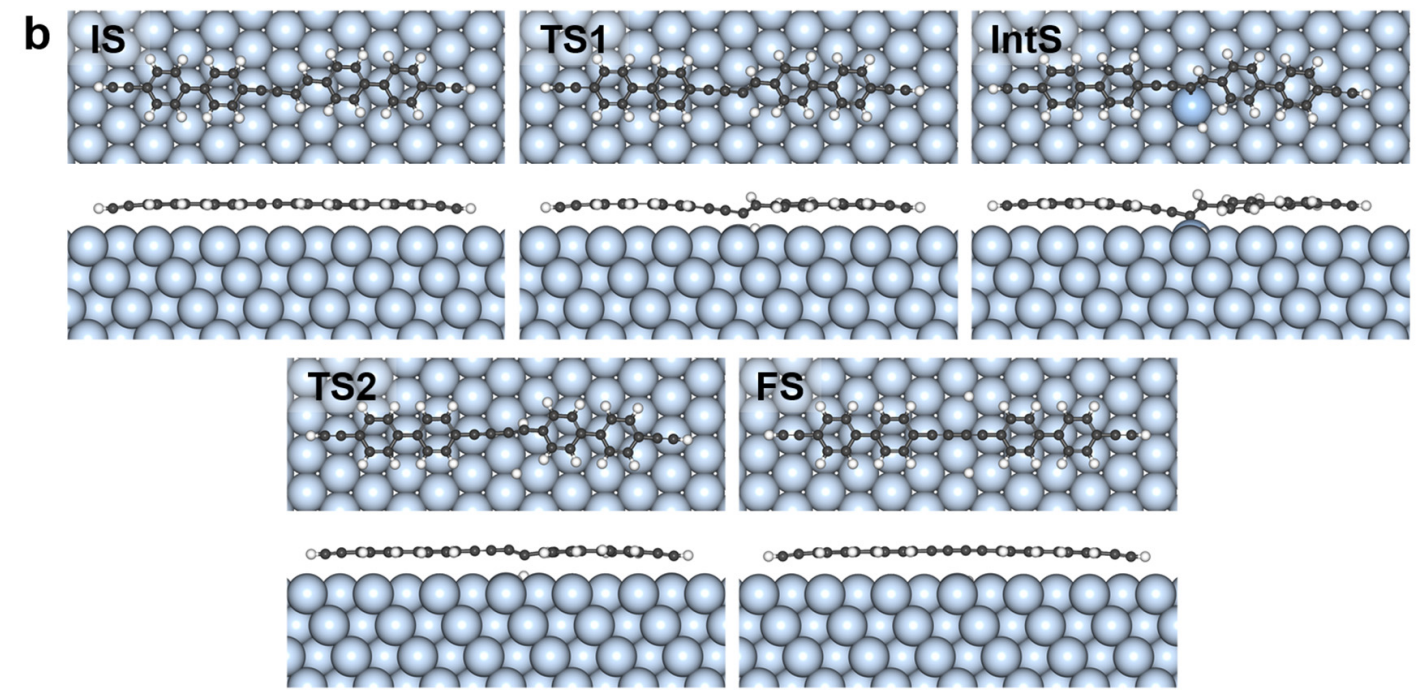

Figure S12. DFT-calculated dehydrogenative reaction pathway from enyne to diyne on $\mathrm{Ag}(111)$. (a) Energy diagram of the reaction process starting from the initial state (IS), through transition state 1 (TS1), intermediate state (IntS), transition state 2 (TS2) to the final state (FS). The relative energies of these states are provided along the pathway with respect to that of the IS. (b) Top and side views of the corresponding structural models. The underlying Ag atom which has direct interaction with the singly dehydrogenated enyne radical (i.e., IntS) is highlighted. C: grey; $\mathrm{H}$ : white; Ag: light blue. 

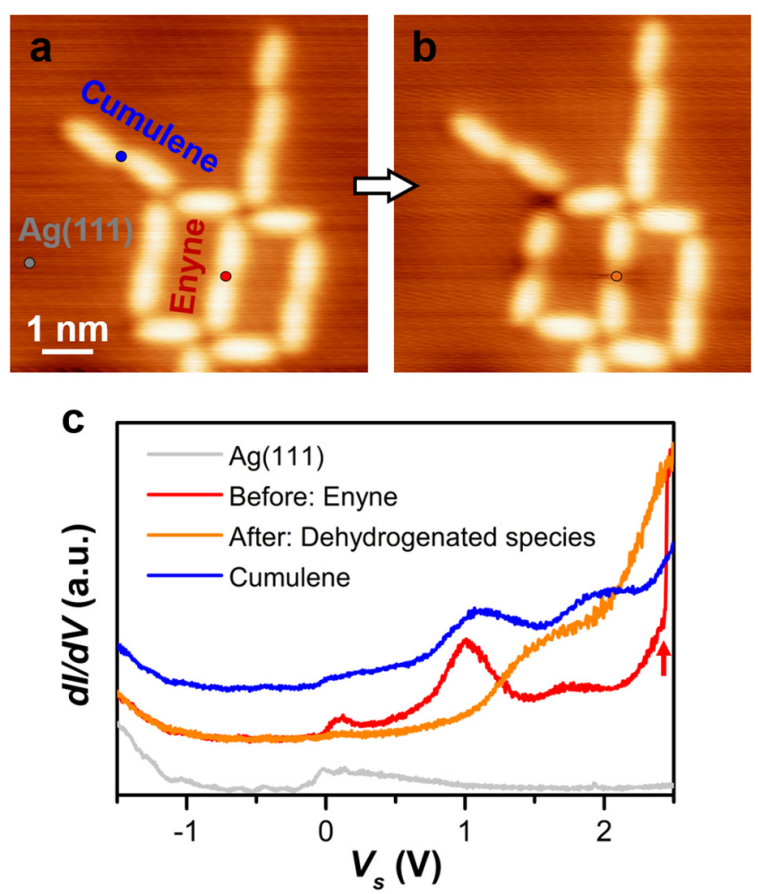

Figure S13. STM images obtained (a) before and (b) after taking the STS spectra. Scanning conditions: $V_{s}=0.1 \mathrm{~V}, I_{t}=1.0 \mathrm{nA}$. (c) STS spectra of enyne, cumulene and dehydrogenated dimers, respectively. The in-situ dehydrogenation starting from enyne can be triggered at a high bias voltage (indicated by the red arrow) simultaneously. 


\section{References}

(1) Ostrowska, S.; Szymaszek, N.; Pietraszuk, C. Selective Dimerization of Terminal Acetylenes in the Presence of PEPPSI Precatalysts and Relative Chloro- and Hydroxo-Bridged N-Heterocyclic Carbene Palladium Dimers. J. Organomet. Chem. 2018, 856, 63-69.

(2) Blöchl, P. E. Projector Augmented-Wave Method. Phys. Rev. B 1994, 50, 17953-17979.

(3) Kresse, G.; Joubert, D. From Ultrasoft Pseudopotentials to the Projector Augmented-Wave Method. Phys. Rev. B 1999, 59, 1758-1775.

(4) Perdew, J. P.; Burke, K.; Ernzerhof, M. Generalized Gradient Approximation Made Simple. Phys. Rev. Lett. 1996, 77, 3865-3868.

(5) Grimme, S.; Antony, J.; Ehrlich, S.; Krieg, H. A Consistent and Accurate ab initio Parametrization of Density Functional Dispersion Correction (DFT-D) for the 94 Elements H-Pu. J. Chem. Phys. 2010, 132, 154104.

(6) Tersoff, J.; Hamann, D. R. Theory of the Scanning Tunneling Microscope. Phys. Rev. B 1985, 31, 805813.

(7) Henkelman, G.; Uberuaga, B. P.; Jónsson, H. A Climbing Image Nudged Elastic Band Method for Finding Saddle Points and Minimum Energy Paths. J. Chem. Phys. 2000, 113, 9901-9904.

(8) Zak, P.; Bołt, M.; Lorkowski, J.; Kubicki, M.; Pietraszuk, C. Platinum Complexes Bearing Bulky NHeterocyclic Carbene Ligands as Efficient Catalysts for the Fully Selective Dimerization of Terminal Alkynes. Chem CatChem 2017, 9, 3627-3631.

(9) Repp, J.; Meyer, G.; Paavilainen, S.; Olsson, F. E.; Persson, M. Imaging Bond Formation between a Gold Atom and Pentacene on an Insulating Surface. Science 2006, 312, 1196-1199.

(10) Repp, J.; Meyer, G.; Stojković, S. M.; Gourdon, A.; Joachim, C. Molecules on Insulating Films: Scanning-Tunneling Microscopy Imaging of Individual Molecular Orbitals. Phys. Rev. Lett. 2005, 94, 026803 .

(11) Gross, L.; Moll, N.; Mohn, F.; Curioni, A.; Meyer, G.; Hanke, F.; Persson, M. High-Resolution Molecular Orbital Imaging Using a $p$-Wave STM Tip. Phys. Rev. Lett., 2011, 107, 086101.

(12) Björk, J.; Zhang, Y. Q.; Klappenberger, F.; Barth, J. V.; Stafström, S. Unraveling the Mechanism of the Covalent Coupling Between Terminal Alkynes on a Noble Metal. J. Phys. Chem. C 2014, 118, 3181-3187.

(13) Wang, T.; Lv, H.; Feng, L.; Tao, Z.; Huang, J.; Fan, Q.; Wu, X.; Zhu, J. Unravelling the Mechanism of Glaser Coupling Reaction on $\mathrm{Ag}(111)$ and $\mathrm{Cu}(111)$ Surfaces: a Case for Halogen Substituted Terminal Alkyne. J. Phys. Chem. C 2018, 122, 14537-14545.

(14) Kong, H.; Zhang, C.; Sun, Q.; Yu, X.; Xie, L.; Wang, L.; Li, L.; Hu, S.; Ju, H.; He, Y.; Zhu, J.; Xu, W. Nickel Adatoms Induced Tautomeric Dehydrogenation of Thymine Molecules on Au(111). ACS nano 2018, 12, 9033-9039. 